\title{
Rechtsgeschichte
}

\section{Alexander Somek}

\section{Das Ganze des Rechts}


leitender Herausgeber der Law Reports, Kommentator der von ihm selbst edierten Urteile im Medium der von ihm herausgegebenen Law Quarterly Review und herausragende Autorität für die Gerichte ( I 69), deren Urteile er wiederum rapportierte.

Jener Diskurs des operativ geschlossenen common law lag nicht mehr allein in den Händen der Richter, sondern auch und vor allem in denen der akademischen Juristen wie F. P. (I 65). Genau aber hielt F. P. diese beiden Sphären auseinander: "Jurists, whether we like it or not, are at the periphery of common law development " (169). Im Zentrum des Rechts operieren die Gerichte. Sie stehen unter Entscheidungszwang. Sollte das common law nicht einfach als willenlose Fortsetzung rechtsexterner Pressionen arbeiten und seiner Autonomie verlustig gehen, bedurfte es einer von Operationszwang befreiten Peripherie, in der die zunehmenden Irritationen der Moderne aufgenommen und in Rechtsform gebracht werden konnten - oder auch nicht. ${ }^{8}$ In der akademischen Peripherie fand ein Sedimentierungsprozess statt, der gesellschaftliche The-

\section{Das Ganze des Rechts*}

I. Die Beschäftigung mit Rechtstheorie ist uncool geworden. Das Fach ist kein Teilgebiet des Wirtschaftsrechts. Zur sozialen Stilisierung juristischer Kompetenz gibt die Theorie nichts her. Im akademischen Bereich ist sie das Steckenpferd des »studentischen Sonderlings «. ${ }^{\mathbf{I}}$

Das Uncoole ist bekanntlich steigerungsfähig. Nicht jeder Schlagersänger ist wie Heino. Nicht jede Theorie ist gleich grau. So genannte »rechtsethische « Debatten über Klonen oder Eu- men auf diejenigen »sound principles of justice " (I42) reduzierte, mit denen die Gerichte dann operieren konnten. Scholarship, in F. P.'s Sprache, is a craft (I 28).

Jede Beobachtung hat ihren Ort. This is not a biography. Der Ort der vorliegenden Studie ist bei einem heutigen akademischen Juristen, der Beobachtungen der viktorianischen akademischen Juristen beobachtet, deren Diskurskonstellationen an Frederick Pollock untersucht und in F. P. chiffriert. Der Ort dieses Buches ist bei der gegenwärtigen englischen Rechtswissenschaft, die sich ein Stück ihrer eigenen Geschichte erzählt - jener Geschichte, die sie selbst verortet: Duxbury ist - wie Pollock - unerhört genau und kenntnisreich. Und auch er ist kein Theoretiker. Jede Beobachtung hat ihren Ort. Ohne weitere theoretische Selbstreflexion kann Duxbury, der Beobachter, nicht sehen, dass er nicht sieht, was er nicht sieht: ${ }^{9}$ dass er eine wunderbare Geschichte der Evolution des englischen Rechtssystems geschrieben hat.

Oliver M. Brupbacher thanasie nehmen sich geradezu populär aus, wenn man sie mit den Dauerbrennern der »allgemeinen Rechtslehre vergleicht. Einem Vortrag, der sich der Frage widmet, was die Ermächtigungsnorm sei, ist das leere Auditorium gewiss. Unter den juristischen Fächern verbreitet die allgemeine Rechtslehre die Aura der unendlichen Langeweile. Die Beschäftigung mit den Grundbegriffen und den Relationen, welche das Rechtssystem ausmachen (287), holt niemanden hinter
8 Niklas Luhmann, Das Recht der Gesellschaft, Frankfurt a. M. I993, 322.

9 Dazu Dirk BAEcker, Kybernetik zweiter Ordnung, in: HeINZ voN Foerster, Wissen und Gewissen. Versuch einer Brücke, hg. von Siegfried J. Schmidt, 2. Aufl. Frankfurt a. M. I994, I7-23, I9.

\footnotetext{
* Andreas Funke, Allgemeine Rechtslehre als juristische Strukturtheorie. Entwicklung und gegenwärtige Bedeutung der Rechtstheorie um I900 (Grundlagen der Rechtswissenschaft, Bd. I), Tübingen: Mohr Siebeck 2004, xii, $338 \mathrm{~S}$. ISBN 3-I 6-I 48476-2
}

\footnotetext{
I Diesen Ausdruck verdanken wir Regina Ogorek. Siehe REgINA OGOREK, Rechtsgeschichte in der Bundesrepublik (I945-I990), in: Rechtswissenschaft in der Bonner Republik. Studien zur Wissenschaftsgeschichte der Jurisprudenz, hg. von Dieter Simon, Frankfurt a. M. I994, I2-99, hier: I4.
} 
dem Ofen hervor. Wer sind uns also Merkel, Bergbohm, Bierling und Somló (20-29)? Sie sind klassische Exempel für Meister der Vergangenheit, über welche die Wirkungsgeschichte ihr Urteil gesprochen hat. Sie sind aus ihr ausgeschieden worden. Ihre Werke sind pedantisch, verschroben, abstrakt und für die Praxis irrelevant.

II. Das Buch von Andreas Funke belehrt uns eines Besseren. Es erinnert daran, dass die so genannte allgemeine Rechtslehre (9-I2) sich historisch als Wegbereiterin der » Rechtstheorie « verstehen lässt (24). Ihre Entstehung markiert den ersten bedeutsamen Versuch, die Voraussetzungen des Sprechens über positives Recht aufzuklären. Eine allgemeine Rechtslehre, die sich als Strukturtheorie des Rechts versteht (9, 39, 78), rekonstruiert die begrifflichen Grundlagen für die juristische Darstellung von Rechtslagen (284). Da juristisches Sprechen auf einen Gegenstand bezogen ist und dieser Bezug die Semantik dieses Sprechens bestimmt, isoliert die diesbezügliche Rekonstruktion die für die Existenz von positivem Recht vorauszusetzenden Grundbegriffe (94). Sie tut dies, indem sie im Hinblick auf empirisches Material allgemeine Schlussfolgerungen auf dessen Möglichkeit zieht.

Der Möglichkeitssinn ist das Markenzeichen der allgemeinen Rechtslehre (etwa 98-99). Sie deckt auf, was sich mit juristischem Sinn (etwa Geboten, Verboten, Erlaubnissen, Ermächtigungen) so alles anstellen lässt. Die Grundbegriffe sind auf Machbarkeit bezogen. Meines Erachtens hätte das niemand klarer ausdrücken können als Kelsen, wenn er die Schadenszufügung bloß unzweideutig als Ermächtigung zur Erzeugung der Schadenersatzpflicht durch den Schädiger gedeutet hätte. ${ }^{2}$ Erst in der Übertreibung zeigt sich der Genius einer Idee. Die allgemeine
Rechtslehre und die aus ihr hervorgehende Rechtstheorie studieren das Ganze des Rechts als ein Kontinuum, in dem sich normativer Sinn generieren lässt. Diese Faszination mit dem Machbaren erklärt auch, weshalb den allgemeinen Rechtslehrern wesentlich war, dass das positive Recht das soziale Gebiet ist, in dem der Wille herrscht (52). Das Augenmerk liegt auf der Setzung von Recht. Nüchternheit ist das Leitmotiv.

III. Funkes Buch ist eine Meisterleistung an Synthese. Anstelle einer langatmigen sukzessiven Wiedergabe von Werken der Altmeister werden deren Fragestellungen, ihre Methoden und ihre Vorstellungen vom Gegenstand ihrer Lehre in kondensierter Form präsentiert (siehe 280-286). Die Auffassungsunterschiede werden aus dem Gemeinsamen herausgearbeitet. Das Unterfangen ist von Sympathie getragen, vor allem, was das positivistische Wissenschaftskonzept angeht. Nach der Lektüre weiß man mehr, nicht nur über das Rechtssystem, sondern auch über die geistige Situation, in der Kelsens Rechtstheorie entstand, auf die man die Entwicklung der allgemeinen Rechtslehre letztlich hinauslaufen sieht.

Wo die Verdienste überwiegen, sollte man nicht beckmesserisch sein. Funkes besonnenes Plädoyer ist lesbar und informativ. Dem Projekt ist bloß die Grenze zu bezeichnen. Funke sieht richtig, dass es den Erfindern der allgemeinen Rechtslehre um das Ganze ging. Sie wollten die Formen isolieren, in denen sich das positive Recht schlechthin präsentiert und vor allem jenen Grundbegriff bestimmen, der enigmatisch dessen Geltungsanspruch signalisiert: das vom moralischen Sollen verschiedene Sollen des Rechts (216-222). Was dieses Sollen bedeutet, sollte freilich bis heute ein Rätsel bleiben. Darüber hinaus wussten die Vertreter der allgemeinen Rechtslehre, dass jede Form sich einer systemati-

2 Kelsen ist alles andere als klar an

diesem Punkt. Siehe Hans KeL-

SEN, Reine Rechtslehre, 2. Aufl.,

Wien I960, II8-II9, 262. 
schen Bestimmung verdankt. Die Rechtsbegriffe sind Teil eines Systems. Was für die Form gilt, bewahrheitet sich auch für den Inhalt. Das Recht ist Ordnung. Aber diese Ordnung, so mag man Funke und seinen Meistern entgegenhalten, ist kein Ding oder Aggregat von Gegenständen der Rechtserkenntnis. Sie ist keine Angelegenheit der theoretischen Feststellung. Die Rechtsordnung als ein System ist eine regulative Idee. ${ }^{3}$ Sie leitet die systematische Konstruktion des Rechts an und hat unterschiedliche Konsequenzen, je nachdem, welches Vorverständnis man davon hat, was eine Rechtsgemeinschaft ausmachen soll. ${ }^{4}$

IV. Das Buch ist durchwirkt von einer immer wiederkehrenden bangen Frage: Ist die allgemeine Rechtslehre als Strukturtheorie zur methodischen Anweisung fähig? Die Frage wird vorsichtig bejaht $(82-84,89,284)$. Gleichwohl fürchte ich, dass die Erwartung, die Rechtstheorie sei zur Überdogmatik berufen, verfehlt ist. Das rechtliche Wissen ist ein Gebiet, auf dem sich die beruflichen Besserwisser tummeln. Im Überfluss der juristischen Expertise kann der Theorie nur eine zutiefst philosophische Funktion zuwachsen. Es ist dies die der Unsinnsvermeidung.
Ich halte die Wahrnehmung dieser Aufgabe für ziemlich cool. Die Rechtstheorie stellt den juristischen Experten den Erhalt ihrer intellektuellen Gesundheit in Aussicht. ${ }^{5}$ Sie verspricht, unseren Verstand und unsere juristischen Begriffe vor den Anschlägen durch die lenkenden, normierenden und urteilenden Stellen zu bewahren. Ich sehe nicht, warum »der Jurist « damit nichts soll anfangen können. Es gibt sogar einen praktisch greifbaren Ertrag. Ohne Begriff von den in Rechtssystemen obwaltenden Relationen sind Juristen in transnationalen Kontexten auf das angewiesen, was die Organe internationaler Organisationen ihnen wortreich und in kreativer Terminologie verabreichen. Ohne einen Sinn für die Problematik der Normativität lässt sich auch nicht der Sinn dafür schärfen, dass immer dann, wenn Normen nichts mehr gelten, im juristischen Denken die Relevanz von Werten und Zweckmäßigkeiten implodiert. Für das Begreifen des intellektuellen Terrains, auf dem sich juristische Sprecher bewegen, ist die Rechtstheorie unverzichtbar. Ohne sie bewegt man sich auf ihm nicht mit intellektuell aufrechtem Gang.

Alexander Somek

\section{Heut' gehn wir ins ... Labor!*}

Nein, heut' geht es nicht ins Maxim! Schenken wir aber Walter Pauly Glauben, so scheint es im »Weimarer Grundrechtslabor « zumindest zeitweise nicht weniger bunt und turbulent zugegangen zu sein. Dem Abgeordneten der Deutschen Volkspartei Rudolf Heinze zufolge steuerte sich in den Grundrechtsberatungen des Verfassungsausschusses das Chaos offenbar selbst: »Ein Gedanke, der augenblicklich auftauchte, wurde in die Verfassung hineingeschrieben. Hatte jemand den Wunsch, einen bestimmten Rechtssatz in der Verfassung festgelegt $\mathrm{zu}$ sehen, so wurde er in die Verfassung hineingenommen, ohne Rücksicht darauf, ob nicht andere Rechtssätze von derselben Bedeutung ebenso gut hätten aufgenommen werden kön-

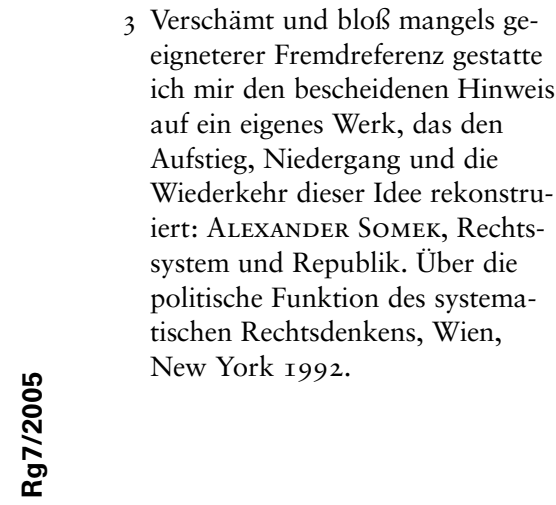

4 Siehe Ronald Dworkin, Law's Empire, Cambridge, Mass. I986, 2 I 2-2 I 3; Christian Hiebaum, Die Politik des Rechts. Eine Analyse juristischer Rationalität, Berlin, New York 2004, 2 I7.

5 Siehe Pierre Schlag, The Enchantment of Reason, Durham, London I998.

\footnotetext{
* Walter Pauly, Grundrechtslaboratorium Weimar. Zur Entstehung des zweiten Hauptteils der Reichsverfassung vom I4. August I9I9, unter Mitarbeit von Olaf Hünemörder, Tübingen: Mohr Siebeck 2004 , VIII, I $22 \mathrm{~S}$., ISBN 3-I 6-I 48479-7
} 